\title{
IDENTIFICATION OF COLLISION MECHANISM AT SEISMOGENIC FAULT INTERFACE USING FINITE ELEMENT ANALYSIS INVOLVING PLATE BENDING APPLICATIONS USING ANT COLONY OPTIMIZATION
}

\author{
P.K. Dutta ${ }^{\mathrm{a}, \mathrm{b},}$ O.P. Mishrab \\ a Erasmus Mundus Post Doctoral Fellow, Faculty of Management and Technological Engineering, University of Oradea, \\ Romania and 3. Assistant Professor, RGMCET, India \\ ${ }^{\mathbf{b}}$ Scientist, Ministry of Earth Sciences, New Delhi, Govt. of India, India
}

Received: 10.12.2016 / Accepted: 10.01.2017 / Revised: 21.04.2017 / Available online: 31.05.2017

DOI: 10.1515/jaes-2017-0002

KEY WORDS: crack-porous; ant colony; geo-dynamical framework; stress-strain; trigger; emergence

\begin{abstract}
:
Understanding space-time correlation in equilibrium matrix when force of collision involving earthquake stress strain interaction. The proposed study shows that non-linear dynamics of earthquake behaviour simulated with ant colony optimization in short timescale deformation by analysis of the stiffness matrix and the stress strain interaction process of the rock pattern. An improved ant colony optimization combined with local search is proposed for solving this complex optimization problem of finding trigger zones for earthquake occurrences. The disturbances at trigger basins for any system cause the collapse of a subsystem leading to stress evolution and slip due to strain nucleation. The stress strain network based on redistribution of stress accumulation are discretized into four states of low stress and strain and a finite element model is established to identify vertices for the stress-strain component and edges for global coupling effects have been constructed for dynamic monitoring of stress and strain behaviour at triggering zones. In this paper, an efficient algorithm is developed for the formation of null basis of triangular and rectangular plate bending finite element models, corresponding to highly sparse flexibility matrices. Triggering basins serve as harbingers of large earthquake where stress-strain interactions have been analyzed by the quasi-static mechanics of seismic precursory stress-strain propagation in the crustal lithosphere. The simulation framework shows that with time, spatial triggering points as stress varies from one point to another to identify the external influences for the body forces and the surface forces for geodynamic frameworks.
\end{abstract}

\section{GENERAL INTRODUCTION}

Understanding earthquake source dynamics is a challenging problem in seismology. The force method of structural analysis in which the member forces are used as unknowns is appealing to engineers since the properties of members of a structure most often depend on the member forces rather than joint displacements. As a result, the force method and some of the advantages it offers in non-linear analysis and optimization has been neglected. A trigger identification system can be done based on the components in the system. An instance being for earthquake faulting is mainly controlled by multi-scales dissipation processes within the fault interface, and by the geometry of pre-existing faults. Conventional models trying to interpret the physical state of the crust before and after earthquake occurrence often fail to explain the associated fault dynamics and stress transfer mechanism involved in seismogenesis (Mishra et al., 2002; Mishra et al., 2003). Firstly, the complex components for dynamics required for understanding fault rupture mechanisms is seen as either having many constraints or so cumbersome that one ultimately loses sight of the original problem of analysis of seismogenesis (Mishra, 2012). The purpose of synchronization of fault segments for seismicity analysis is not to explain every detail of an evolutionary process of interaction between the stress and strain components, but its fundamental behaviour analysis of finding origin of the quake. Models imitating the complex interplay of the fluctuations in local dynamics and long range interactions for any time series (Abe et al., 2005, Varotsos et al., 2006) for fault networks has long been of interest in the geodynamic framework of fault dynamics. Our first hypothesis is that geodynamic framework can be made analogous to a twodimensional interacting fault network based dynamic system undergoing quasi static evolution. Uniform distribution of seismic cohesive forces is non-existent in fault networks as because of the three dimensionality of fault system (Zhao et al., 2002; Mishra and Zhao, 2003).Earthquakes occurrence is attributed due to slippage when accumulation of strain exceeds the bearing capacity of the rock. When such causative factors exist earthquakes occur due to slight increase in stresses under the influence of external factors disturbing the equilibrium which acts as a trigger. The force method of structural analysis requires the formation of a maximal set of independent self-equilibrating stress systems (SESs), known as a null basis. The elements of this basis form the columns of a matrix, $B$, known as the self-stress matrix. In order to obtain a statically determinate structure, known as the basic (released or primary) structure of $S$, the constraints corresponding to redundant are removed. Consider the joint loads vector where $n$ is number of entries of the applied nodal load vector and let $r$ denote the $m$-dimensional vector of generalized independent element forces. The equilibrium conditions of the structure can 
then be expressed as $\mathrm{A}$ is an $\mathrm{n} \times \mathrm{m}$ equilibrium matrix with self stress matrix with sparse flexibility. In order to obtain a statically determinate structure, known as the basic structure of $\mathrm{S}$, the constraints corresponding to redundant are removed. Consider the joint loads vector as $\mathrm{p}=\left\{\mathrm{p}_{1}, \mathrm{p}_{2}, \ldots \mathrm{p}_{\mathrm{n}}\right\}^{\mathrm{t}}$ where $\mathrm{n}$ is number of entries of the applied nodal load vector and let $r$ denote the m-dimensional vector of generalized independent element forces. Each column of B is called a self-equilibrating stress matrix (null basis matrix). A maximal set of SESs (null vectors) is known as a statistical basis (null basis). Minimizing the complementary potential energy requires that $r$ minimize the quadratic form $1 / 2 \mathrm{r}_{\mathrm{t}} \mathrm{F}_{\mathrm{m}} \mathrm{r}$ where $\mathrm{F}$ is an $\mathrm{m} \times \mathrm{m}$ block diagonal

element flexibility matrix and $G=B_{1}^{t} F_{m} B_{1}$ and redundant are denoted by vectors $\mathrm{q}=\left\{\mathrm{q} 1, \mathrm{q} 2 \ldots \mathrm{q} \Psi_{(\mathrm{s})}\right\}$ where $A r=\mathrm{p}$ where $\mathrm{n}$ being the number of entries and $\mathrm{r}$ denote the $\mathrm{m}$ dimensional vector whereby $r=B_{0} p+B_{1} q$. The equilibrium conditions of the structure can then be expressed as distant triggering of small fault slips (Gomberg et al. (1997) by moderate earthquakes have been reported through various studies. The degree of cohesiveness in the rock assemblage varies inversely with pores and cracked percentage in the total volume of rock and the behaviour of stress - strain generation changes with fluid contents of the cracked volume of rock matrix (Singh et al. (2011). The necessity to analyze continuous non-consecutive cellular automata exhibiting self- organized critically, demonstrates the generality of the phenomenon (Varotsos et al. (2005).We test this mechanisms through cellular automation model in (Dutta et al., 2012) to validate long range elastic interaction and short range precursory behaviour for the geodynamic system in chapter 3.Many works (Stein, 1999; Scholz, 2002) based on investigations for static or dynamic stress transfer has been well reviewed on the fault system dynamics and their long range interactions that have found to exhibit strong indications of synchrony in fault behaviour (Scholz, 2010) in the stress-slip based interaction framework. An alternative explanation can be provided by cascade (Sarilis et al. (2009) model that explains the seismic nucleation which manifests increasingly larger sub-events: a large earthquake occurs when a smaller earthquake triggers a cascade of increasingly larger slip events. A series of questions can be analyzed in identifying the processes that lead to either positive or negative feedbacks to perturbations based upon static and/or dynamic stress changes. In societal conflict with any unrest a section of the population will take part in the event while others are not; so as a big event is occurring a deviation or drift will always occur, in earthquake monitoring the offset fault rupture displacement. The main aim of the work is to investigate the emergence of trigger events in hierarchical fault systems (Dominguez et al., 2012) due to variation of seismicity patterns through evolutionary optimization technique of ant colony framework. The idea of the seismogenic crust to have seismic zones or basins that trigger large motions is a conclusive evidence of the phenomena conducive stress accumulates in critical state (Evison and Rhoades, 2004; Scholz, 1991) based on acoustic emissions for seismic electrical signals (Varotsos and Alexopoulos, 1984 a, b; Varotsos et al., 1993).The proposed mechanism involves the interaction of three processes: initialization parameters for the ants, ants build solutions to update the pheromone and the ant identifies the unique path to the maxima or minima existing within the stress-strain framework. It is assumed that every ant has knowledge of the global coordinating plane and has two functions for $n$-dimensional system as the ant moves in terms of two coordinates for exploration and exploitation in search of the global minima for rest. An agent ant or any particle in terms of strategy will follow the knowledge in three paths or heuristics based on position, fitness value which is found based on a generic algorithm and velocity. The ant moves along the pile model is to be estimated along a line or at a finite difference dependence of triggering delays on different system conditions. In the case of impulsive loading the initial conditions will involve discontinuities of stress behaviour along certain boundaries as a possible explanation for the widespread occurrence of space-time long-range correlations in earthquakes dynamics. Bankwitz (1980) has deduced that the energy of earthquakes is radiated to a significant part from relatively small, numerous fault planes and not from large single faults whereby evolution of pre-seismic slip to seismic rupture requires understanding the evolutionary dynamics of the local and global correlated framework of stress-strain behaviour whose impact on the overall organization of the underlying tectonic strata can be neither assessed nor understood in isolation. The proposed article aims at the modelling of a geodynamical framework capable of explaining geo-tectonic and societal conflicts using mathematical properties of emergence in complex systems (Crucitti et al. (2004), Varotsos et al. (2005). By correlating the ant colony (Dorigo et al. (1996) framework with fault dynamics behaviour, projecting cascading failure by the inherent path seeking activity of ants as agents can define the fault dynamics for dynamic stresses and strains for space and time in large geodynamic networks for accumulated strain deformation. Fault behaviour and stress drop in critical conditions can be studied by the typical features of self organizing dynamics and local heterogeneity for evolution of stress. Heterogeneity of the spatio-temporal process of the system is the tendency of the system to maintain the condition of stability in the active state of dynamic equilibrium. The global coupling effect on the near neighbourhood blocks is really significant as the process reflects long range interactions. In this paper, the geodynamic interacting system is validated by means of ant colony interaction framework for spatial identification of trigger basin. The basic ant clustering algorithm and its improved algorithm with define the stress force and optimize the stress force per unit displacement for the rock matrix. For this purpose we work on stiffness matrix k. As we know that the stiffness matrix represents kij as the force required for producing unit displacement at $\mathrm{j}$. If we consider the rock as matrix of two degrees of freedom for a node, we can model a structure whereby the stiffness matrix for a certain load clusters. to solve the non-convergence problems and it is found that the clustering effect. Improved ant colony algorithm incorporated in the study uses a genetic algorithm-based improvements based on the added factor of variation, mutation, and thus faster convergence. If the value of the sample is less than pheromone threshold value $\mathrm{q}$, then we take the maximum of pheromones as a path. If there is more than the maximum phermone, the same maximum value from a randomly selected as a path. When we calculated deviated error for each sample to its corresponding Euclidean distance of the cluster centers and MIN the better the clustering effect. Calculated values of ants to find the minimum value MIN, the best path to the path corresponding to the value-oriented iterations. 


\section{ANALYSIS OF TRIGGERING BASED ON PHYSICAL MODELS OF EARTHQUAKE OCCURENCE}

In recent years, the research area in the identification of the stress strain interaction process is to critically identify the null vectors whereby the forces in the previous generators should be zero, after removal of the generating edges of all the double edges from the interface graph, the remaining null vectors should be selected from the remaining interface graph. This mechanism in the stress strain process identifies the global behaviour evolution and emergence from the many relations of simple behaviours (Cornfold et al., 2004). The complex system analysis can be mapped as an evolving graph model with trigger points representing realistic deformation due to stress strain deformations equivalent to physical entities for vertices of the graph, and the edges of the graph (infinite two dimensional lattices) evolving over time under the action of forces. This evolutionary system has a series of critical states and a number of endogenous and exogenous behaviour patterns (Dutta et al., 2012) which give a contrasted opinion about the feasibility of earthquakes prediction from impossibility (full chaos) to probabilistic long term prediction (weak chaos). Both earthquake occurrence and societal conflict behaviour follows the same path of accumulation of stress followed by strain accumulation through relaxation path. The spatial distribution of faults in lithosphere is an extremely complex dynamic system from the point of view of both its structure and processes therein (Turcotte, 1992; Korvin, 1992; Kagan, 1994; Keilis-Borok, 1994). Hainzl et al.(2000) showed that relative seismic quiescence of seismicity spring-block system with transient creep characteristics deals with consistent empirical precursory observation of stress strain interactions appear prior to events that might help to develop a cellular automation model of Lu and Ding,1993. The predictability of catastrophic events is based on work by (Sornette,2002) who proposed that underlying cause of catastrophic failure can be sought months and even years before the abrupt catastrophic event in the buildup of stress for a cascade failure like an accelerating rise of a offset as the nearest neighbours interact in normal system (Laughlin and Joannopoulos,1978). A complex system is driven away from equilibrium by observing interacting components for the system which is likely part of accumulation of strain can be analyzed by the offset value at the perimeters of the changing system or the fault rupture offset (Oglesby, 2008).The offset can be considered as the break in the system dynamics. When an entity appear, a vertex will appear in the graph, when a communication will be established between two entities, an edge will appear between the two corresponding vertices. The complex system analysis can be mapped as an evolving graph model with trigger points representing deformation zones equivalent to physical entities for vertices of the graph, and the edges of the graph (infinite two dimensional lattices) evolving over time under the action of forces. Plates and membranes have discontinuous change in the crosssectional area at section of stress discontinuity. Force extension relation neglecting elastic strains helps consider material as rigid for piece wise linear force extension is $\mathrm{F}=\mathrm{F} 0+\lambda(\mathrm{t})$ where $\mathrm{F}$ is the membrane force at any instant on the cross-section normal to the plane of motion. In the absence of body forces the equation of motion of an isotropic elastic medium are satisfied if displacement is given by $\mathrm{q}=\operatorname{grad} v+\operatorname{rot} \mathrm{A}$, where $v$ and $\mathrm{A}$ satisfy the wave equation The sand-pile evolutionary system has a series of critical states and a number of endogenous and exogenous behaviour patterns (Mishra et al.2007 a, 2007b) which identify quasi-periodic events in earthquake model (Figure 1). Devising a lattice model of sand in a rotating cylinder for equations to model sand pile dynamics and ripple formation for sand pile relaxation a situation where reorganization of a maxima and minima within the pile dominates the flow. This self organization for sand pile for slow rotation or tilt driving occurs in the form of rotation and deposition. In order to analyze the flow start searching with ' $n$ ' ants for optimum value in search space for randomness through rules which constantly change based on initial conditions. Initialize ant position and movement in positive and negative direction for correlation. In order to apply the ACO algorithm to a specific problem, it is necessary to represent it as a set of different paths for ants to travel. In the problem of finding sparse null basis, different sequence of generators is considered as a tour for an ant to travel, therefore the cooperative ant agents search to find the best generator sequence resulting in a sparse null basis. Since both the edge numbering and its order in the generator sequence are important, the pheromone amount is specified by two indices where the index $i$ is the generator order in the set of generators, and the index $j$ shows the edge number. As an example, the amount of pheromone for selection of the edge number 5 as the 2 generator in the generators set. In our algorithm, first $m$ artificial ants are initially positioned on $m$ edges of elements as primary generators, and then ACS algorithm is applied. Stress state in jth site and if $\epsilon$ is the longitudinal strain change in length in the plane of motion $\lambda$. Force required by the membrane for its yielding will depend on boundary displacement and boundary tractions known under strain states and stress tensor given by the two-dimensional coordinates. The triggering will have an impact velocity which affects the yield stress and strain hardening rate. The stress strain network can be interpreted by the increase in steady-state energy transmitted to the surface acting as a source of redistribution of energy into the earth tectonic framework. A fault system is a simple one dimensional framework of a chain of blocks interacting in the local level globally coupled in a rigid plate. The behaviour of the system at jth site as $\mathrm{F}(\mathrm{j}, \mathrm{t}+1)=\mathrm{fl}(\mathrm{f}(\mathrm{j}, \mathrm{t})+$ local interaction + global coupling effects. Slip motions of the block change at seismo-dynamic equilibrium condition disturbs balance of the system which depends on the dynamic network of stress-strain relations. When the stress of the block exceeds the value of a threshold value or maximum friction then the block slips to a new position.

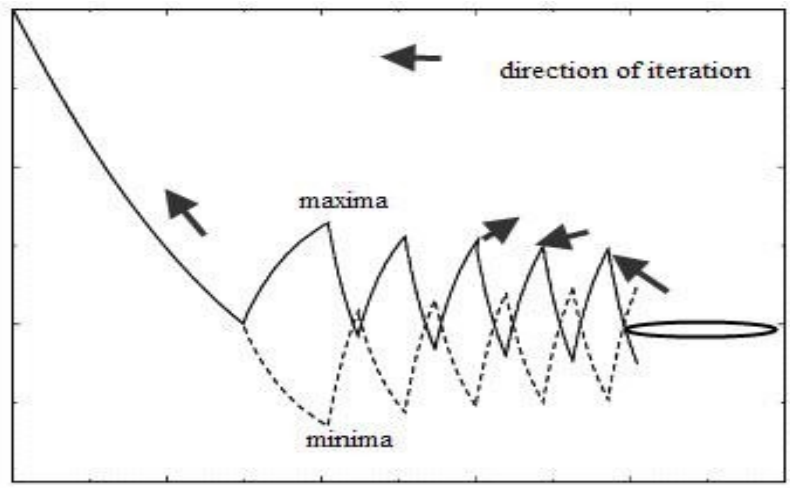

Figure 1. Sand grain drop model followed by avalanche and deposition 


\section{ANALYSIS OF TRIGGERING BASED ON GEODYNAMIC FRAME WORK}

Geodynamic Monitoring (GDM) as specified by (Godzikovskaya and Strom, 2007; Outkin and Yurokov, 2009) represents a predictive system design for studying the intensedeformed condition of separate blocks of an earth's crust based on occurrence of deterministic modelling of natural and dynamic processes. When stress is applied to the material at elevated temperatures, creep occurs by a process of dislocation movement or simply stress directed diffusion of vacancies in the crystal lattice (Mishra et al., 2008) which affects the natural time series analysis for critical phenomena in sand pile model (Varotsos et al., 2010; Varotsos et al., 2011). Difference between potential and rupture energy equivalent to stress accumulated strain is physically measurable $u i$ is change of displacement $\delta \mathrm{u}_{i j}$ is change of stress associated $\mathrm{S}$ is the spherical surface of radius source. Boundary displacements or boundary tractions are known under strained state and stress tensor will be given by Eulerian coordinates for strain tensor may also be referred under the same system $\tau \mathrm{ij}=\lambda \delta$ uij $11+$ $2 \mu \mathrm{Cij}$. Diffusional creep depends on re-distribution of local vacancy concentrations subjected to normal stress behaviour. When a vacancy is formed at the boundary subjected to a tensile stress, $\sigma$ a force $\sigma \mathrm{b} 2$ tends to move distance, $b$; where $b 3$ is directly proportional to $\Omega$ the atomic volume (Varotsos and Alexopolous, 1977; Varotsos et al., 1978). $\eta$ is the amount of pheromone deposited on the generator number $s$ as a candidate for being the $r$ th generator. It is assumed that there is an equal amount of pheromone is the set of generators that remain, to be chosen by ant $k$ as the $r$ th generator and

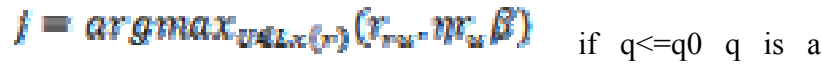
random vector distributed in $[0.1) \mathrm{q} 0$ is the parameter $0 \leq \mathrm{q}_{0} \leq 1$ and $\mathrm{J}$ is the random variable.

$P_{\mathrm{rs}} \quad \Gamma_{\mathrm{rs}} \eta_{\mathrm{rs}} \beta / \sum \mathrm{u} \in \mathrm{L}_{\mathrm{k}}(\mathrm{r}) \Gamma_{\mathrm{ru}} \eta_{\mathrm{ru}}$ deposited initially on each generator. After an ant chooses one edge as a generator, the local updating rule on that chosen generator is performed in order to shuffle the solution and prevent focusing on a specific solution. The local updating rule modifies the amount of pheromone by once all the ants complete their own tours, the pheromone will be updated for all the edges according to the global updating rule. This pheromone updating is intended to allocate a greater amount of pheromone to shorter tours. The rule is given by the following equation: $D$ is the sparsity coefficient of the globally best tour (number of non-zero elements in the selected null basis) and $0<\beta<1$ is the pheromone decay parameter. The best ant tries to find the sparse null basis. Pre-slip patterns are linked to parameter changes (structural perturbation) responding as anomalies and a single triggering function driven by rock permeability changes induced by crustal deformations (Caracausi,2009). In an earthquake, these zones strain in response to both the total stress (due to co-seismic compliant zone weakening) and co-seismic stress change (due to permanent compliant zone weakness). Steady state framework for stress-strain interaction at random trigger basins based on increasing transitions is shown in Figure 2. The correlated trigger basins for seismic basins for seismic evolution based near neighbourhood search for increasing energy transitions is shown in Figure 3.

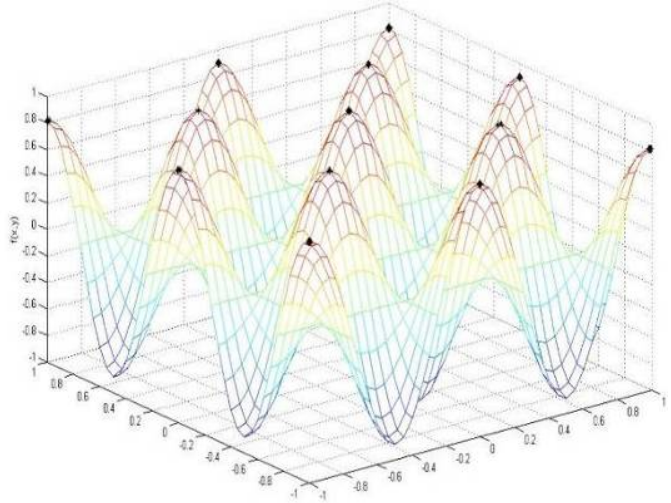

Figure 2. Steady state framework for stress strain interaction at random trigger basins based on increasing energy transitions

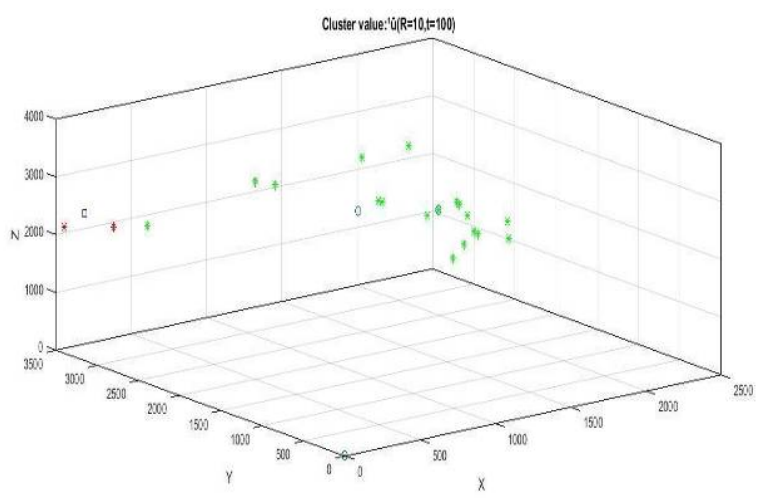

Figure 3. Experimental results for Steady state framework for stress strain interaction at random trigger basins

\section{SYSTEM MODEL WITH SIMULATION}

The goal of this chapter is to describe the contribution of ant colony based algorithm in the understanding of the lithosphere dynamics and the unique challenges to map connectivity of basins based on emergent properties, multiple equilibrium, path dependence, and nonlinearities inherent in the bound system that has been ignored (Rial et al 2004).Ant Colony Optimization (ACO) is an algorithm used for solving the shortest path problem which is inspired by real ants and included in meta-heuristic algorithms (Dorigo \& Stützle, 2004).Ant colony behaviour is found to emerge periodically in swarms and establish perimeter barriers involving interactions among individual agents to study processes of collective action. In the ant colony clustering algorithm each data object is represented with an agent whereby the corresponding ant in the colony has an activity space corresponding to a two dimensional grid. Ant calculates the similarity of the nodes and those within the viewing radius. In the process of clustering analysis ant picks up the node most dissimilar to the entire neighbourhood by decision to pick and drop elements to the neighbourhood. The analysis is done in the following manner whereby the initial cluster centers are chosen randomly as the hills in the sand pile as in Figure 1. Where the size of the pheromone on the path ij represents the pheromone evaporation coefficient, on behalf of the coefficient of residual pheromone for the increase of the pheromone,

$$
\tau_{i j}=\left((1-\rho) t_{i j}\right)
$$


In the problem of finding sparse null basis, different sequence of generators is considered as a tour for an ant to travel, therefore the cooperative ant agents search to find the best generator sequence resulting in a sparse null basis. Since both the edge numbering and its order $\mathrm{n}$ the generator sequence are important, the pheromone amount is specified by two indices where the index $i$ is the generator order in the set of generators, and the index $j$ shows the edge number. As an example, $\beta$ shows the amount of pheromone for selection of the edge number 5 as the 2 generator in the generators set. In our algorithm, first $m$ artificial ants are initially positioned on $m$ edges of elements as primary generators, and then ACS algorithm is applied as follows: An ant $k$ chooses the $r$ th generator by applying the rule of the following equation. An ant colony system is developed for the formation of sparse null basis leading to sparse self-stress matrices, and correspondingly highly sparse flexibility matrices for triangular and rectangular plate bending finite element models is the amount of pheromone deposited on the generator number $s$ as a candidate for being the $r$ th generator. It is assumed that there is an equal amount of pheromone is the set of generators that remain, to be chosen by ant $k$ as the $r$ th generator and deposited initially on each generator. It is the corresponding heuristic value which remains constant throughout the iterations and, unlike pheromone amount, is not modified. After an ant chooses one edge as a generator, the local updating rule on that chosen generator is performed in order to shuffle the solution and prevent focusing on a specific solution. The local updating rule modifies the amount of pheromone by all the ants complete their own tours, the pheromone will be updated for all the edges according to the global updating rule. This pheromone updating is intended to allocate a greater amount of pheromone to shorter tours. D is the sparsity coefficient of the globally best tour (number of non-zero elements in the selected null basis) and 0 $<\beta<1$ is the pheromone decay parameter. The best ant tries to find the sparse null basis. Each ant choices are determined according to the size of the amount of pheromone on the path depending on the constraints. The constraint is a dependency between the genes, which is solved by using an encoding which replace this global constraint (all genes different) by a local constraint to pick up other similar item in its neighbourhood current_cluster_number else if suit $(\mathbf{0})==\mathbf{0}$; expand radius until suit $(\mathbf{o})==1$, save location $\mathbf{p}$ and radius into ant a's memory, drop the item o. Slip motion of block changes equilibrium of the global stress field that disturbs balance for fault network through pheromone trails in the global network. The pheromone trials can have a total of $\mathbf{2 4}$ or $\mathbf{1 6}$ values depending on the combination of compressive and extensive stress and strain respectively between $\mathbf{0}$ and $\mathbf{1}$. In order to understand the suitability of a complex adaptive system approach, the graph based geodynamical framework model is proposed to interpret the interaction between pair wise stress-strain effects for environmental decision making involved in complex system dynamics. In the proposed work an effort has been made to map the steady-state increase of stress strain behaviour in a complex dynamics (as in Figure 2) and find the non random trigger basin in hyper-structures as a result of neighbourhood interactions. This method to analyze long range correlations and coupling effects in the inherent stress-strain field identifies steady state stress accumulation and distribution. Ants after the completion of the search are mobile according to the rules for the next search. After the completion of the cycle, the ants will find the optimal solution for the transfer. Pheromone will be more for ants that choose the shorter path, the amount of pheromone on the short path will lead to high concentrations of pheromone more ants choose this branch, eventually all the ants are concentrated to this branch. In the proposed study avoid selected paths by decreasing their pheromone level and it works well in face of large scale problems using non-linear ant system which employs the concept of non linearity from teach automata field to prevent the algorithm from early convergence. Transient dynamic stress spreads homogeneously with adjustments connected to static stress changes. Global correlation or static stress/strain effects play a significant role in the generation of earthquakes. Simulation features the use of dynamic spatial patterns to explore the dynamics of complex systems for representation of spatial processes and spatial interaction. The processes related to the earthquake generation are characterized by some internal dynamical structure that are not completely random (Rundle and Klein, 2000; Matcharashvili and Ghlonti, 2002). Each ant starts with the same search radius, and then automatically adjusts the neighbourhood radius around its position and memorizes the size of the pure neighbourhood during the clustering process. Zones for finding ants are attracted are covered by pheromones and maintained. As the zone is less affected ant pheromones leave a trail which allows other ants to follow. This is similar to earthquake domain pattern where aseismic slip becomes predictive. Ants as agents when once in energetic mode particle interactions like repulsion and friction (Helbing et al., 2000). If the new position of slip is better than the original position, then replace with the new location, for the original position; or retain the original position as slip change. There are several functions to determine how much pheromones get left behind based on the distance travelled as studied by (Rooijackers, 2005) through ant colony optimization. A selection of random basins taken at equidistant points where stress strain interaction in the dynamic precursory model. The natural system behaviour is the analysis of the steady state changes. Search step size should be reduced with the increase of the number of iterations in order to be able to get a more accurate solution search. The iteration method reveals better learning coefficient for the dynamic model (Figure 4). This pheromone trial is naturally related with the proposed correlation measures around local neighbourhoods. The rate of increase or drop and rise of elastic energy involved in the process through multi-agent based study reflects dynamical regime of seismic processes by calculation of their measurable characteristics is important in the search for possible earthquake predictive dynamical markers for trigger basins placed at equidistant intervals (Figure 2) in effect of global and local stress field. The study proposes a system of ant colony framework in relation to global correlation interaction with precursory phenomena shows that nearest neighbour faults will be negatively coupled and positive coupling will occur only with more distant faults as shown in Figure 3. Once in the pure neighbourhood, we again perform near neighbourhood iteration for initializing cluster centers $\mu 1 \ldots, \mu \mathrm{k}$; based on mode and then re-compute cluster centers (means of data points in cluster).Select modes as the initial cluster centers . Compute the region in the grid balance where the most pure and tallest and dense cluster is located. This is a region which may trigger the earthquake occurrence in the future. Neighbourhood radius is the size of a pure neighbourhood for the search of a trigger function calculated with the following routine while Suite $T(0)=0$.In actual cases the global coupling effects can be negative as shown by the reduced activity of earthquakes at near and far earthquake sources. Enhanced clustering activity is observed at points far 
away after ' $\mathrm{n}$ ' time period showing that steady state trigger basins have an active impact in the global field of stress evolution and slip nucleation at spatial points which interact among themselves. Fast global optimization with fast searching rate and high optimizing precision spatial existence of seismogenesis has a critical value of stress and strain condition that can be evaluated in both discrete and continuous attributes.

\section{RESULTS OF SIMULATION AND DISCUSSION}

Optimization involves analysis of the stress strain components for a self organizing framework where performing solution components removed as iteration solution as shown in Figure 4. The improved algorithm has the following parameters.

pls $=0.1 ; \%$ local optimization threshold pls (equivalent mutation rate)

solution_temp $=$ zeros $(\mathrm{L}, \mathrm{N}+1)$;

$\mathrm{k}=1$;

while $(\mathrm{k}<=\mathrm{L})$

solution_temp $(\mathrm{k},:)=$ solution_ascend $(\mathrm{k},:)$;

$\mathrm{rp}=\operatorname{rand}(1, \mathrm{~N}) ; \%$ produces a $1 * \mathrm{~N}(51)$ random-dimensional array, for $\mathrm{i}=1: \mathrm{N}$

if $\mathrm{rp}$ (i) $<=\mathrm{pls} \%$ less than a value of the random pls change the corresponding path identifier

current_cluster_number $=\operatorname{setdiff}([1: \mathrm{K}]$, solution_temp $(\mathrm{k}, \mathrm{i})$; $\operatorname{rrr}=\operatorname{randint}(1,1,[1, \mathrm{~K}-1])$;

change_cluster = current_cluster_number (rrr); solution temp $(\mathrm{k}, \mathrm{i})=$ change cluster;

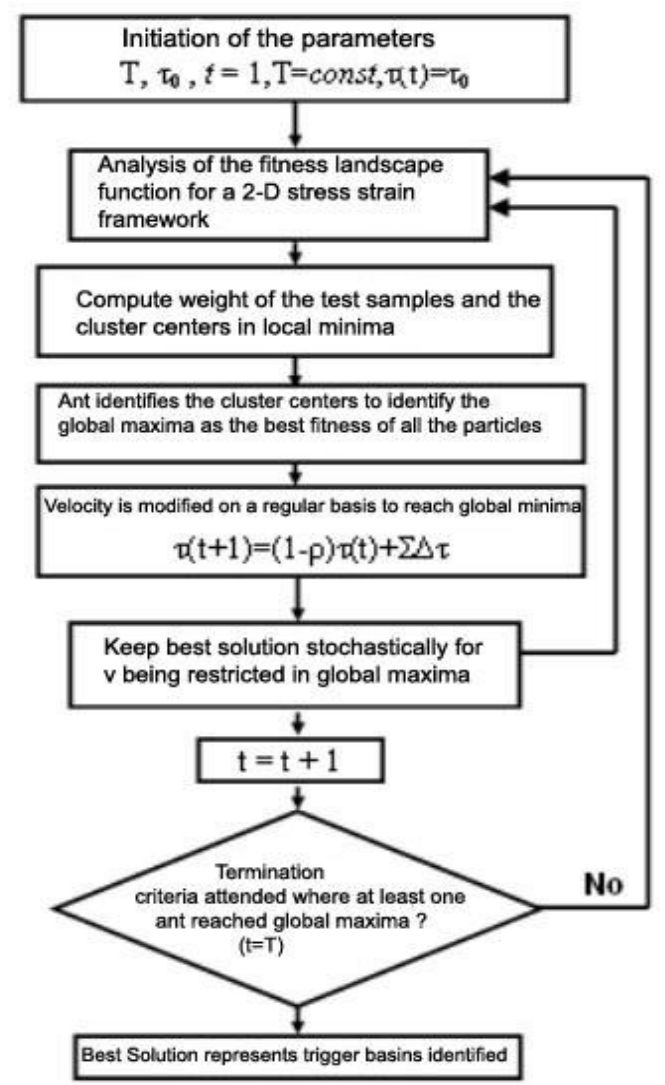

Figure 4. Ant Colony Procedure for spatial clustering in a nonlinear ant system
Largest strain changes that occur at the last stages of failure and the amplitude or size of the strain change are related to nucleation patch moment release. The results strongly outline that using long- term radon monitoring and stress analysis for interpretation of the self organizing networks that interact at the triggering basins. Simulations prove that there is a close proximity of local dynamics and long range interactions having a connection with stress evolution both on grounds of the projections and experimental observations conducted by (Varotsos et al., 2013). Natural time series analysis conducted by (Varotsos et al., 2002; Varotsos et al., 2003 a, b). It was found that a characteristic change of seismicity is observed around a measuring site at which precursory signals, termed Seismic Electric Signals activities (Varotsos et al., 1991) are emitted which is simultaneous with a similar change identified at remote distances, i.e., more than around $1000 \mathrm{~km}$. Similar changes of seismicity have been also recently found in Sarilis et al. (2013). The time for the formation of the null basis matrices is, in general, higher compared to the graph theoretical methods, however since the meta-heuristics are rapidly progressing and becoming more and more efficient, one hopes such algorithms will become superior in the very near future.

\section{REFERENCES}

Abe, S., Sarlis, N.V., Skordas, E. S., Tanaka, H. K., and Varotsos., P. A. (2005), Origin of the usefulness of the naturaltime representation of complex time series. Physical review letters 94, 17: 170601.

Bak, P. and Tang, C. (1989), Earthquakes as a self-organized critical phenomena, Journal of Geophysics Research, 94, 15635-15637.

Bankwitz, P. (1980). Zum Bewegungsablauf an Bruchst6rungen mit seismotektonischer Aktivit it. Z. Geology Wissez. (Berlin), 8: 353-362.

Burridge, R., and Knopoff, L. (1967), Model and theoretical seismicity. Bulletin of Seismological Society of America.57, 341-371.

Caracausi, A., Italiano, F., Martinelli, G., Paonita, A. and Rizzo, A. (2009), Long-term geochemical monitoring and extensive/compressive phenomena: case study of the Umbria Region (Central Apennines, Italy). Annals of Geophysics, 48(1): doi: 10.4401/ag-3178.

Crucitti, P., Latora, V., Marchiori, M. (2004), Model for cascading failures in complex networks. Physics Reviews E 69: 045104 .

Cornford, D., Opper, M., Shawe-Taylor, J., Roulstone, I., and Clark, P., (2004), Variational Inference in Stochastic Dynamic Environmental Models, Avalaible online: personal.maths.surrey.ac.uk/st/I.Roulstone/VISDEM_outline.pdf.

Dutta, P.K., Mishra, O. P., Naskar, M.K. (2012), A Poisson Process Hidden Markov Cellular Automata Model in Earthquake Genesis and Conflict Analysis: A Physical 
Approach. Journal of Seismology \& Earthquake Engineering, 14(2).

Dominguez, R., Tiampo, K., Serino, C.A., Klein, W. (2012), Characterizing Large Events and Scaling in Earthquake Models with Inhomogeneous Damage. Geophysical Monograph Series 196:41-54.

Dorigo, M., Maniezzo, V., and Colorni, A. (1996), Ant System: Optimization by a Colony of Cooperating Agents. In: IEEE Transactions on Systems, Man, and Cybernetics-Part B: Cybernetics, 26(1).

Dorigo, M., and Stützle, T. (2004), Ant colony optimization. Cambridge, Mass: MIT Press.

Godzikovskaya, A. and Strom, A.L. (2007), Specific features of seismological investigations in regions of hydraulic structures. Power Technology and Engineering 30(12), 705-711, doi: 10.1007/ BF02447461.

Gomberg, J., Blanpied, M.L., Beeler, N.M. (1997), Transient Triggering of Near and Distant Earthquakes. Bulletin of the Seismological Society of America, Vol. 87, No. 2, pp. 294-309.

Hainzl, S., Z"oller, G., Kurths, J., and Zschau, J. (2000), Seismic quiescence as an indicator for large earthquakes in a system of self-organized criticality. Geophysical Research Letters, 27(5), pp. 597-600.

Helbing, D., Farkas,I. and Vicsek, T. (2000), Simulating dynamical features of escape panic. Nature 407:487-490.

Kagan, Y.Y. (1994), Observational evidence for earthquakes as a nonlinear dynamic process, Physica, D77, p 160-192.

Keilis-Borok, V.I., 1994, Symptoms of instability in a system of earthquake-prone faults, Physica, D77. pp. 193199.

Korvin, G. (1992), Fractal Models in the Earth Sciences, Elsevier, 396 pp. 1-17.

Laughlin, R.B., and Joannopoulos, J.D. (1978), Effect of second-nearest-neighbor forces on the vibrations of amorphous SiO2. Physics Reviews B 17, pp. 2790-2792.

Matcharashvili, T. and Ghlonti, E. (2002), Detecting differences in dynamics of small earthquakes temporal distribution before and after large events, Computers \& Geosciences 28, 693-700.

Mishra, O. P., Zhao, D., Kayal, J. R., Reena, D. E., \& Singh, O. P. (2002), Tomography of the Source Area of the 2001 Bhuj Earthquake: Evidence for fluids at the hypocenter. Geophysics. Research. Letters, 24, 501-504.

Mishra, O.P. and Zhao, D. (2003), Crack density, saturation rate and porosity at the 2001, Bhuj, India earthquake hypocenter: a fluid driven earthquake? Earth Planet Science Letters, 212, pp.393-405.

Mishra, O. P., Zhao, D., Umino, N., Hasegawa, A. (2003), Tomography of northeast Japan forearc and its implications for interpolate coupling. Geophysics Research Letters, 30, DOI: $10.1029 / 2003$ GLO17736.
Mishra, O. P. and Zhao, D., (2004), Seismic evidence for dehydration embrittlement of the subducting Pacific slab. Geophysical research letters, 31(9), L09610.

Mishra O.P. (2004), Lithospheric heterogeneity and seismotectonics of NE Japan Forearc and Indian regions, unpublished D.Sc. thesis, Ehime University, Japan, pp.223.

Mishra, O. P., Kayal, J. R., Chakrabortty, G.K., Singh, O. P., and Ghosh, D. (2007a), Aftershock investigation in Andaman Nicobar of the 26 December 2004 earthquake (Mw 9.3) and its seismotectonic implications. Bulletin Seismological Society America, 97 (1A), S71 - S85.

Mishra, O. P., Chakrabortty, G.K., Singh, O. P., Kayal, J. R., and Ghosh, D. (2007b), Aftershock investigation in Andaman - Nicobar Islands: An antidote to Public Panic. Seismological Research Letters, 78 (6), pp.591 - 600.

Mishra, O. P., Zhao, D., and Wang, Z. (2008), The genesis of the 2001 Bhuj, India, earthquake (Mw 7.6): A puzzle for peninsular India. Journal Indian Minerals Special Issue, 61 (34) \& 62 (1-4), $149-170$.

Mishra, O. P., Zhao, D., Ghosh, C., Wang, Z., Singh, O. P., Ghosh, B., and Gaonkar, S. G. (2011), Role of crustal heterogeneity beneath Andaman-Nicobar Islands and its implications for coastal hazard. Natural hazards, 57(1), pp. 5164

Mishra, O. P. (2012), Seismological Research in India. Proceedings of Indian National Science. Academy Publication (PINSA), 76 (3), $361-375$.

Oglesby, D. (2008), Rupture Termination and Jump on Parallel Offset Faults, Bulletin of the Seismological Society of America; 98(1)- 440-447; DOI: 10.1785/0120070163.

Outkin, V.I. and Yurkov, A.K. (2009), Radon as a Deterministic indicator of natural and industrial geodynamic processes. Doklady Earth Sciences, Vol: 427:833-836, doi:10.1134/S1028334X09050274.

Rhoades, D.A., Robinson R., Gerstenberger, M.C. (2011), Long-range predictability in physics- based synthetic earthquake catalogues. Geophysical Journal International.Vol 185, pp. 1037-1048. doi: 10.1111/j.1365-246X.2011.04993.x.

Rial, J.A. (2004), abrupt climate change: Chaos and order at orbital and millennial scales. Global and Planetary Change, 41, 95-109.

Rooijackers, M., (2002), K-means clustering with Ant colony optimization $\quad 24^{\text {th }}$ July,2011 www.es.ele.tue.nl/education/ci/5ci10/clusterants.pdf.

Rundle, J. and Klein, W. (2000), Geo-Complexity and the physics of earthquakes, Geophysical Monograph Series, 120 AGU- 147-163, Washington.

Sarlis, N. V., Skordas, E. S., Lazaridou, M. S., \& Varotsos, P. A. (2008). Investigation of seismicity after the initiation of a seismic electric signal activity until the main shock. In: 
Proceedings of the Japan Academy. Series B, Physical and biological sciences, 84(8), pp.331.

Sarlis, N. V., Skordas, E. S., Varotsos, P. A., Nagao, T., Kamogawa, M., Tanaka, H., \&Uyeda, S. (2013). Minimum of the order parameter fluctuations of seismicity before major earthquakes in Japan. Proceedings of the National Academy of Sciences, 110(34), pp. 13734-13738.

Scholz, C.H. (1991), Earthquakes and faulting: selforganized critical phenomena with a characteristic dimension, in Spontaneous Formation of Space-Time Structures and Criticality, Kluwer Academic Publishers, Netherlands, pp. $41-56$.

Scholz, C. H. (2002), The Mechanics of Earthquakes and Faulting, Cambridge University Press, Cambridge.

Scholz, C.H. (2010), Large Earthquake Triggering, Clustering, and the Synchronization of Faults Bulletin of the Seismological Society of America, Vol. 100, pp. 901909, doi: 10.1785/0120090309.

Singh, A. P., Mishra, O. P., Rastogi, B. K., and Kumar, D. (2011), 3-D seismic structure of the Kachchh, Gujarat, and its implications for the earthquake hazard mitigation. Natural Hazards, 57(1), pp.83-105.

Sornette, D. (2002), Predictability of catastrophic events: Material rupture, earthquakes, turbulence, financial crashes, and human birth. Proceedings of the National Academy ofSciences. Vol. $\quad 99, \quad$ pp. 25222529 doi:10.1073/pnas.022581999. Available online at $<\mathrm{http}: / /$ www. pnas.org/ content/99/suppl.1/2522.full.Retrieved29thJuly, 2011.

Stein, R. S. (1999), the role of stress transfer in earthquake occurrence, Nature 402, no. 6762, pp. 605-609.

Varotsos, P. and Alexopoulos, K. (1977), Calculation of the formation entropy of vacancies due to anharmonic effects, Physical Review B 15, pp. 4111-4114.

Varotsos, P., Ludwig, W., and Alexopoulos, K. (1978), Calculation of the formation volume of vacancies in solids, Physical Review B 18, 2683-2691.

Varotsos, P., and Alexopoulos, K. (1984), Physical properties of the variations of the electric field of the earth preceding earthquakes, I, Tectonophysics 110.1: 73-98.

Varotsos, P., and Alexopoulos, K. (1984), Physical properties of the variations of the electric field of the earth preceding earthquakes, II. Determination of epicenter and magnitude, Tectonophysics 110, pp. 99-125.

Varotsos, P., Alexopoulos, K. and Lazaridou, M. (1991), On recent seismic electrical signal activity in nothern Greece, Tectonophysics 188, 403-405.

Varotsos, P., Alexopoulos, K., and Lazaridou, M. (1993), Latest aspects of earthquake prediction in Greece based on Seismic Electric Signals II, Tectonophysics224, pp. 1-37.
Varotsos, P. A., Sarlis, N. V., and Skordas., E. S. (2002), Longrange correlations in the electric signals that precede rupture. Physical Review E, 66- 1, 011902.

Varotsos, P. A., Sarlis, N. V., Skordas, E. S. (2003a), Longrange correlations in the electric signals that precede rupture: Further investigations, Phys. Rev. E, 67, 021109 (13).

Varotsos, P. A., Sarlis, N. V., Skordas, E. S. (2003b), Attempt to distinguish electric signals of a dichotomous nature, Phys. Rev. E. 68, 031106 (7).

Varotsos, P. A., Sarlis, N. V., Tanaka, H. K., and Skordas., E. S. (2005), Similarity of fluctuations in correlated systems: The case of seismicity.Physical Review E 72, no. 4 : 041103.

Varotsos, P. A., Sarlis, N. V., Skordas, E. S., Tanaka, H. K., and Lazaridou, M. S. (2006), Attempt to distinguish long-range temporal correlations from the statistics of the increments by natural time analysis. Physical Review E 74, no. 2: 021123.

Varotsos, P. A., et al. (2010), Natural-time analysis of critical phenomena: The case of seismicity. EPL (Europhysics Letters) 92.2 (2010): 29002

Varotsos, P., Sarlis, N. V., Skordas, E. S., Uyeda, S., \& Kamogawa, M. (2011), Natural time analysis of critical phenomena. Proceedings of the National Academy of Sciences, 108(28), pp, 11361-11364

Varotsos, P. A., Sarlis, N. V., Skordas, E. S., \& Lazaridou, M. S. (2013), Seismic Electric Signals: An additional fact showing their physical interconnection with seismicity. Tectonophysics, 589, pp. 116-125.

Turcotte, D. (1992), Fractals and Chaos in Geology and Geophysics, Cambridge University Science 1-17, pp.398. 